\title{
Customer satisfaction e brand competition
}

\author{
ANTONIO IAZZI
}

\begin{abstract}
Obiettivo del paper: il lavoro propone un modello di valutazione della customer satisfaction che consenta di: a) disporre di indicatori di sintesi della soddisfazione del consumatore; b) acquisire informazioni analitiche ed esaustive dello stesso costrutto, utili per la definizione delle strategie di marketing c) verificare l'esistenza di legami tra la soddisfazione e i comportamenti conseguenti, con un approfondimento specifico sul ruolo espletato dal brand.

Metodologia: analisi descrittiva e multivariata sui dati raccolti tramite un test condotto, con questionario strutturato, su un campione non probabilistico di 350 consumatori che acquistano jeans.

Risultati: il lavoro ha consentito di: a) individuare le determinanti della soddisfazione del consumatore di jeans; b) valutare l'esistenza di una relazione lineare tra la soddisfazione e comportamenti successivi all'esperienza di consumo del prodotto; c) verificare l'assenza del predetto legame in talune relazioni di fedeltà, nello specifico, e nel caso di acquisto del "Top brand of the mind".

Limiti della ricerca: il campione oggetto di indagine non permette una generalizzazione dei risultati.

Implicazioni manageriali: il lavoro si propone di fornire informazioni utili per l'implementazione di percorsi di customer satisfaction e la definizione delle strategie di marketing nel settore del jeans.

Originalità del lavoro: la metodologia adottata offre un contributo per la costruzione di un modello di consumer knowledge management in grado di supportare la definizione delle strategie di marketing $e$, in particolare, la ricerca della differenziazione.
\end{abstract}

Parole chiave: customer satisfaction; comportamenti intenzionali; marketing management

Purpose of the paper: this paper proposes a model for customer satisfaction measurement, able to: a) provide a brief overview on customer satisfaction indicators; b) suggest detailed information thorough, which could be useful in order to; c) verify the existence of any connection between customer satisfaction and intentional behaviors, with an in-depth analysis on the role of brand.

Methodology: descriptive and multivariate analysis. Data have been obtained through a structured questionnaire, administered to a non-probabilistic sample made up of 350 consumers who buy jeans.

* Ricercatore di Economia e Gestione delle Imprese - Università degli Studi del Salento e-mail: antonio.iazzi@unisalento.it

sinergie, rivista di studi e ricerche

n. 92, Settembre-Dicembre 2013, pp. 183-205

ISSN 0393-5108 - DOI 10.7433/s92.2013.10
Ricevuto: 31/08/2013

Revisionato: 13/09/2013

Accettato:07/10/2013 
Results: findings allowed to: a) find out customer satisfaction determinants in the jeans sector; b) observe the existence of a linear relation between satisfaction and post purchase behavior; c) verify the essence of the aforesaid connection in terms of loyalty and, in particular, in reference to top-of-mind brands.

Limitations: the sample does not allow the generalization of the obtained results.

Managerial implications: the work intends to provide useful information in terms of customer satisfaction measurement and marketing strategies implementation in the jeans sector.

Originality: the methodology adopted offers a contribution to the construction of a model of consumer knowledge management able to support the definition of marketing strategies and, in particular, the search for differentiation.

Keywords: customer satisfaction; intentional behaviours; marketing management

\section{Introduzione}

L'analisi del comportamento del consumatore assume, nel contesto attuale, una maggiore significatività, alla luce delle dinamiche evidenziate dai mercati globali. Varietà e variabilità dei modelli di consumo impongono ai marketing manager una specifica attenzione nell'analisi ed interpretazione del modo in cui la value proposition dell'impresa è percepita sui mercati di riferimento. Risulta quindi indispensabile attivare modelli di customer satisfaction in grado di monitorare le dinamiche di consumo e consentire, quindi, l'adozione di strategie di marketing in grado di generare competitività nel lungo termine. Ciò in considerazione dell'impatto che l'esperienza di consumo può avere sui comportamenti successivi alla stessa.

I recenti contributi della letteratura hanno evidenziato il ruolo espletato dalla soddisfazione sia sui comportamenti del consumatore (tra gli ultimi, Dimitriades, 2006; Chi e Qu., 2008; Faullant e Matzler, 2008), che sulla redditività dell'impresa (Luo e Homburg, 2007) e sulla determinazione del valore per gli azionisti (Luo et al., 2010; Raithel et al., 2012).

Gli elementi ricorrenti sull'argomento concernono (Giese e Cote, 2000):

- la specificità della soddisfazione quale risposta ad un processo di valutazione effettuato;

- i caratteri del processo con cui si attiva la risposta (confronto tra aspettative, standard di raffronto e esperienze di consumo);

- la validità temporalmente definita dei risultati ottenuti.

Per quanto attiene la misurazione della soddisfazione, in letteratura si rileva la presenza di un numero modesto di proposte di scale di valutazione (per le scale mono-item si rammentano quelle proposte da Westbrook, 1980; Hausknecth, 1988; Spreng et al., 1996; una scala multi-item è proposta da Oliver, 1997, rispetto al corpus teorico presente (Guido et al., 2010), a cui si sono affiancati modelli teorici che hanno provato a misurare la performance del prodotto rispetto a singoli attributi (importance-performance analysis e modello di Kano). 
Con riferimento ai settori di applicazione, l'analisi della letteratura rileva un elevato ricorso allo studio della customer satisfaction nel settore terziario e nelle relazioni di tipo business-to-business (Hirsh, 2011; Brock et al., 2013); viceversa, negli ultimi anni sono rare le applicazioni nel settore industriale, nonostante all'interno dello stesso vi siano contesti ad alta intensità competitiva, quali quelli del "Fashion", per i quali si ritiene particolarmente utile il ricorso all'analisi del costrutto in esame nell'ambito dei processi di decision making.

Considerando gli sviluppi della letteratura sul tema, il lavoro propone un modello di valutazione della customer satisfaction nel settore dei jeans che consenta di: a) disporre di indicatori di sintesi della soddisfazione del consumatore; b) acquisire informazioni analitiche ed esaustive dello stesso costrutto, utili per la definizione delle strategie di marketing c) verificare l'esistenza di legami tra la stessa soddisfazione e i comportamenti conseguenti, con un approfondimento sui principali "Top brand of the mind" emersi dall'indagine.

La scelta del settore del jeans è motivata dai caratteri dello stesso (varietà di produzione, orientamento alla differenziazione, crescita dei volumi di consumo) e dalle continue e specifiche attenzioni manifestate da parte della letteratura manageriale, sebbene impegnata esclusivamente nello studio dei processi di acquisto del consumatore (tra gli altri, si vedano Lee, 1990; Park e Lee, 1999; Delong et al.,2002; Vrontis e Vrontis, 2004; Wu, 2005; Wu e Delong, 2006; Jin et al., 2010). Tale constatazione evidenzia la specificità dello stesso ambito competitivo nel più ampio settore fashion, determinando, di conseguenza, la necessità di un approfondimento dei comportamenti post-acquisto.

Al fine di perseguire i predetti obiettivi si è pianificato un approccio di tipo mixed method (con adozione quindi di diverse metodologie), articolato sui seguenti step: a) indagine on desk sulla letteratura manageriale che si è occupata del settore in esame e indagine qualitativa, tramite focus group, entrambe finalizzate alla individuazione degli item della soddisfazione; b) somministrazione di un questionario (la cui ultimazione è avvenuta in seguito ad un test pilota condotto su 20 consumatori abituali di jeans) ad un campione non probabilistico di consumatori che acquistano abitualmente jeans ed elaborazione dei dati con tecniche statistiche di natura sia descrittiva che multivariata - utilizzando il software SPSS - per verificare l'esistenza di correlazioni significative tra le variabili oggetto di analisi.

Le informazioni così acquisite appaiono indispensabili per la definizione delle strategie di marketing di quei settori che evidenziano dinamiche competitive complesse, anche per via della componente emozionale che accompagna l' acquisto e della forte differenziazione dell'offerta.

\section{I fondamenti teorici di riferimento}

La comprensione della soddisfazione del consumatore riveste un ruolo di particolare rilievo nell'ambito degli studi di marketing management. Tradizionalmente, l'interesse della letteratura per lo studio della soddisfazione del 
consumatore è stato dettato dalla consapevolezza delle possibili conseguenze che da essa potevano scaturire per l'impresa (tra gli altri, Spreng et al., 1996). Negli ultimi anni il rinnovato interesse sul tema, confermato in particolare dalla numerosità degli studi che si sono occupati del costrutto in esame, ha riguardato: i) l'applicazione di modelli di customer satisfaction in prevalenza nel settore dei servizi e nelle relazioni di tipo business-to-business; ii) la valutazione della influenza manifestata nei confronti del comportamento del consumatore; iii) la misurazione dell'impatto sulle performance aziendali e sul valore per gli azionisti.

Così come sostenuto in premessa, il panorama degli strumenti di misurazione della soddisfazione appare contenuto rispetto al corpus teorico proposto dalla letteratura. In particolare, le scale di soddisfazione proposte considerano il costrutto in esame quale esito di una valutazione che ha per oggetto la performance del prodotto. Tra le scale mono-item si rammenta quella ancora utilizzata e proposta da Westbrook (1980), con la quale si chiede al consumatore di esprimere un giudizio attraverso la scala del differenziale semantico, composta da una coppia opposta di aggettivi (solitamente di natura emotiva, data la prevalenza di tale componente nella percezione della soddisfazione; Westbrook e Oliver, 1991). Tra le scale multi-item vi è quella proposta da Oliver (1997): 12 item, per i quali gli intervistati sono chiamati ad esprimere il proprio livello di accordo o disaccordo.

In parallelo, si sono sviluppati modelli teorici che, muovendo dalla considerazione che il consumatore è in grado di valutare i singoli caratteri del prodotto acquistato, misurano la soddisfazione in considerazione della performance di uno o più attributi dello stesso. Un modello classico è quello matriciale dell'analisi importanza-performance (la prima elaborazione è da ascrivere a Martilla e James, 1977) il quale prevede la valutazione del grado di importanza e del livello di performance rilevati per un insieme di attributi opportunamente selezionati. Esso rappresenta un utile strumento di diagnosi (Johns, 2001; Matzler et al., 2003) che consente al management di: i) individuare le aree che necessitano di interventi di miglioramento (Sampson e Showalter, 1999); ii) effettuare una efficace mobilitazione e distribuzione delle risorse a favore delle aree di maggiore interesse (Levenburg e Magal, 2005). Gli sforzi compiuti di recente dalla letteratura vanno nella direzione di colmare alcune criticità del modello (Matzler et al. 2004, Chen, 2014), determinate dal fatto che nella matrice gli attributi contribuiscono al giudizio complessivo in modo lineare, senza considerare che ciascuno potrebbe contribuire in misura diversa ed, altresì, che in talune circostanze l'importanza può essere influenzata dal livello di performance (soprattutto se non si assicurano adeguate prestazioni).

Tongue e Moore (2007) hanno proposto un'alternativa che considera la valutazione del grado di importanza attribuita in fase di scelta e del livello di soddisfazione (sempre rispetto a determinati item), modello invero già adottato nel settore pubblico (Tanese et.al. 2003). Nell'ambito del presente lavoro la matrice rappresenta uno dei due strumenti utilizzati per l'interpretazione dei risultati, unitamente al monitoraggio dell'indicatore di soddisfazione media ponderata con l'importanza attribuita dal consumatore a ciascun item all'atto dell'acquisto. 
L'ultimo ambito di indagine riguarda il grado di influenza della soddisfazione sui comportamenti del consumatore. L'attenzione del management sui comportamenti successivi all'esperienza di consumo è indispensabile nell'analisi del risultato delle politiche aziendali, poiché hanno un indubbio impatto sulle performance aziendali (una rassegna è proposta da Luo e Homburg, 2007). La letteratura ha individuato i due seguenti comportamenti consequenziali: i) l'intenzione di un nuovo acquisto (tra i primi contributi si ricorda Labarbera e Mazursky, 1983); ii) l'intenzione di consigliare l'acquisto del brand ad altri (quale effetto della fiducia maturata, così come descritto, tra gli altri, da Costabile, 2000).

Sebbene non vi sia una condivisione unanime della letteratura, alcuni studi (Fullerton, 2005; Fornell et al., 2006, Rauyruen e Miller, 2007, Tsai et al., 2006) hanno dimostrato l'esistenza di un intenso legame tra la soddisfazione e l'intenzione di riacquisto. È da rilevare altresì che la soddisfazione rappresenta una condizione necessaria ma non sufficiente per generare fedeltà; essa alimenta la fiducia che il cliente via via matura e conseguentemente la probabilità che il comportamento dello stesso cliente si reiteri nel tempo, generando fedeltà (Castaldo, 2002; Costabile et al., 2004). È stato verificato (Yi e La, 2004; McMullan e Gilmore, 2008) che, in presenza di fedeltà (al prodotto o al brand), singole esperienze non soddisfacenti di consumo potrebbero non compromettere l'intenzione di reiterare l'acquisto.

\section{II modello concettuale e la metodologia della ricerca}

In considerazione di quanto rilevato in precedenza, il modello concettuale proposto è strutturato considerando due aree (fig. 1): la prima concerne la misurazione della soddisfazione e del legame della stessa con i comportamenti postacquisto; la seconda è quella dell'interpretazione dei risultati della soddisfazione, utile per individuare le aree potenziali di miglioramento (indicatore medio ponderato della soddisfazione e adozione della matrice importanza-soddisfazione).

Fig. 1: Il modello di analisi della customer satisfaction

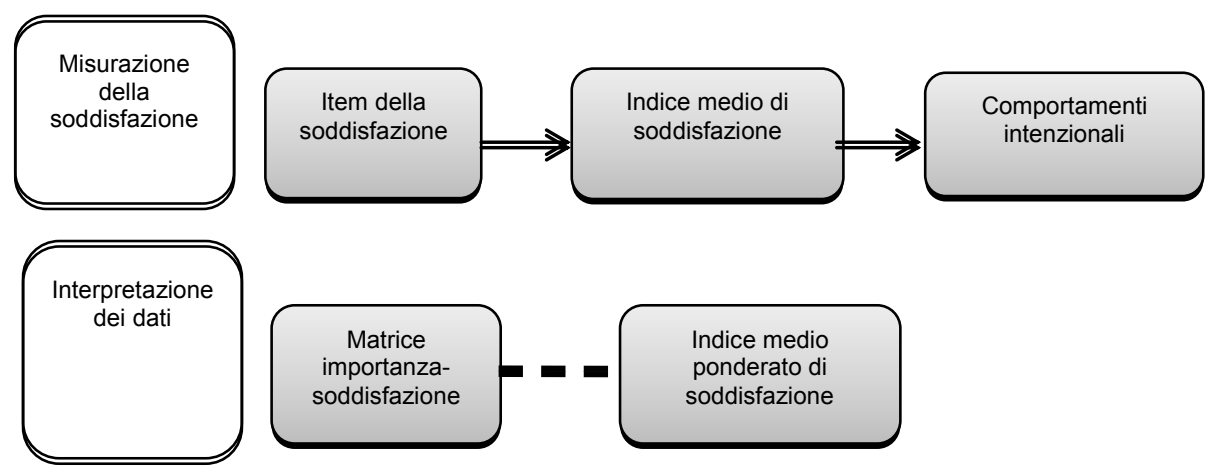

Fonte: ns. elaborazione 
In particolare, il calcolo dell'indicatore di soddisfazione media ponderata con l'importanza attribuita a ciascun item consente di attribuire un diverso peso al livello di soddisfazione ottenuto in considerazione, appunto, del ruolo attribuito all'item in fase di scelta. L'analisi della matrice importanza-soddisfazione, così come sostenuto in precedenza, permette di individuare le aree di maggiore criticità e quindi contribuire ad un miglior impiego delle risorse aziendali nella ricerca della soddisfazione del cliente.

Il primo step di lavoro, così come descritto in premessa, ha riguardato l' individuazione degli item della soddisfazione per il prodotto jeans, con i quali costruire il questionario. A tal fine si sono realizzate due attività: a) una indagine on desk sulla letteratura manageriale da cui non sono emersi contributi significativi sul tema della soddisfazione nel settore in esame; si è ritenuto pertanto utile effettuare una analisi della letteratura sulle determinanti di acquisto del prodotto (Young, 1980; Workman, 1988; Enochs, 1993; Delong, 1998; Chowdhary, 2002; Delong et al., 2002; Herbst e Burger, 2002; Zhang et al., 2002 Jin et al., 2010; Rahman, 2011); b) un'indagine on field, di tipo qualitativo, condotta tramite focus group su 15 consumatori

Avendo individuato gli item, si è proceduto con la costruzione del questionario necessario per il secondo step di lavoro e composto da due parti. La prima dedicata alla verifica dei dati personali dei rispondenti, con riferimento tanto alle generalità che alla propensione degli intervistati ad acquistare il prodotto. La seconda riservata a: i) la verifica dell'importanza che i differenti caratteri del prodotto assumono nel processo di scelta del consumatore (utile per l'adozione della matrice importanzasoddisfazione e per il calcolo dell'indice di soddisfazione medio ponderato); ii) la misurazione della soddisfazione del consumatore per il brand che acquista con maggiore frequenza; iii) la verifica delle future intenzioni comportamentali. La versione finale è frutto anche dei risultati di un test pilota condotto su 20 consumatori .

Per la misurazione delle variabili d'analisi è stata utilizzata una scala di misurazione standard ( $1=$ valore minimo; $5=$ valore massimo $)$. I dati raccolti sono stati elaborati con tecniche statistiche di natura sia descrittiva che multivariata (con l'ausilio di SPSS). In particolare queste ultime hanno consentito di verificare l'esistenza di correlazioni significative tra la soddisfazione percepita per i singoli item e l'indice complessivo di soddisfazione, nonché tra quest'ultimo e i comportamenti consequenziali del consumatore.

La somministrazione del questionario, effettuata tra marzo ed aprile del 2013, ha coinvolto un campione di 350 consumatori che acquistano jeans, di età compresa tra 18 e 50 anni, in maggioranza donne ${ }^{1}$. La disponibilità di spesa media non supera $\mathrm{i}$

L'assenza di report di settore, così come comunicato, tra gli altri, dalla Federazione Sistema Moda Italia, e la conseguente carenza di informazioni in merito ai caratteri della popolazione di riferimento, non hanno consentito la costruzione di un campione in linea con i caratteri di uno specifico universo. Pertanto, si è data prevalenza alla popolazione 
70,00 euro nel $57,5 \%$ dei rispondenti, percentuale che raggiunge 1 ' $83,7 \%$ se si eleva il limite a 100,00 euro. Si consideri altresì che il 37,2\% degli intervistati acquista, in media, un nuovo paio di jeans ogni cambio di stagione, il $29,8 \%$ una volta l'anno e il 20,9\% ogni 2-3 mesi.

\section{I risultati dell'indagine empirica}

\subsection{La misurazione della soddisfazione e delle conseguenze comportamentali}

Gli item individuati per la misurazione della soddisfazione del consumatore di jeans (primo step del lavoro) sono i seguenti: comfort, vestibilità, design/modello, versatilità d'uso (caratteristiche funzionali); colore, lavaggio, qualità del tessuto (caratteristiche tecniche), rifiniture, novità modello, contemporaneità del modello, brand, prezzo, valori del brand, originalità lavaggio (caratteristiche simboliche).

La verifica dell'affidabilità interna degli item impiegati per la costruzione del questionario è avvenuta utilizzando il coefficiente $\alpha$ di Cronbach, il quale fornisce una misura della coerenza interna dei gruppi di item. Il risultato ottenuto, pari a 0,823 , pone in evidenza un'elevata correlazione tra gli item del questionario (consistenza interna alta); pertanto, si può sostenere che ciascun item offre un reale contributo alla misura della soddisfazione in esame e nell'insieme tutti gli item si riferiscono allo stesso costrutto.

Una seconda verifica effettuata concerne la validità del criterio, ovverosia la capacità degli item di misurare il costrutto in esame. Quindi, l'insieme degli item che si intendono proporre e validare e la misura sintetica del fenomeno in esame (indicatore medio di soddisfazione) devono essere associati e correlati. È stata, perciò, effettuata un'analisi di correlazione tra la variabile "Soddisfazione", comprendente tutti i 14 item osservati e la "Soddisfazione complessiva". Si fa presente che il livello di soddisfazione complessiva rilevato (è stata posta una domanda diretta all'intervistato, chiedendo di esprimere un giudizio sul grado di soddisfazione) è pari a 4,13 , con oltre l' $82 \%$ delle risposte distribuite tra i valori 4 e 5.

Il coefficiente $r$ di Pearson è positivo e presenta un livello di significatività pari a $0,015(<0,05)$, consentendo di affermare che la correlazione tra le variabili in esame è significativa e che le due variabili covariano, ovvero ad alti valori di una variabile corrispondono alti valori dell'altra variabile e viceversa.

Avendo constatato l'esistenza della relazione tra la soddisfazione per i singoli item osservati e la soddisfazione complessiva, si è proceduto rilevando i valori medi di soddisfazione rispetto a ciascun item considerato e la deviazione standard.

femminile $(60 \%)$ dati il coinvolgimento e la maggiore sensibilità tradizionalmente dimostrati nei confronti dell'acquisto di prodotti dell'abbigliamento (Auty e Elliot, 1998). 
Fig. 2: La misurazione della soddisfazione

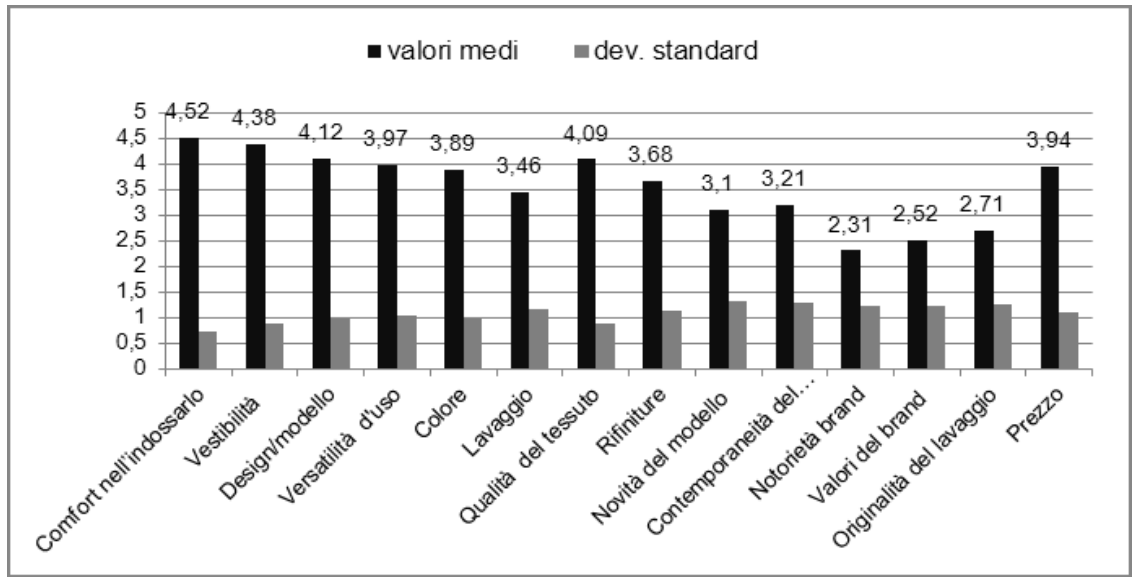

Fonte: ns. elaborazione

"Comfort" (valutazione media 4,52), "Vestibilità" $(4,38)$ e "Design/modello" $(4,12)$ sono gli attributi che contribuiscono in modo considerevole alla soddisfazione del consumatore. Anche in tale circostanza, la bassa varianza è frutto di risposte vicine al valore medio. L'ultima fase del percorso di sviluppo degli item di misurazione, che consente di perseguire il secondo obiettivo del lavoro, concerne la verifica del legame esistente tra la percezione della soddisfazione nelle esperienze di consumo e i comportamenti intenzionali futuri del consumatore. Tale verifica, definita di validità del costrutto (De Vellis, 1991), si riferisce alla capacità degli item di fornire una misura, nello specifico, della soddisfazione che sia coerente con $\mathrm{i}$ fenomeni ad essa collegati, che nel caso specifico sono da ascrivere all'intenzione di riacquistare il prodotto/brand e all'attivazione di passaparola positivo.

L'analisi di correlazione (Chi Square Test di Pearson) ci consente di valutare l'eventuale esistenza di un legame tra la soddisfazione e le tre variabili appena citate.

Tab. 1: L'analisi di correlazione di Pearson (Chi Square Test) tra la soddisfazione e i comportamenti intenzionali

\begin{tabular}{|c|c|c|c|}
\hline \multicolumn{2}{|c|}{} & Intenzione di acquisto & Passaparola \\
\hline \multirow{2}{*}{ Soddisfazione } & Pearson Correlation & $0,140^{*}$ & $0,270^{* *}$ \\
& Sig. (2-tailed) & 0,022 & 0,000 \\
\hline \multirow{2}{*}{ Intenzione di acquisto } & Pearson Correlation & 1 & $0,330^{* *}$ \\
& Sig. (2-tailed) & & 0,000 \\
\hline \multirow{2}{*}{ Passaparola } & Pearson Correlation & $0,330^{* *}$ & 1 \\
& Sig. (2-tailed) & 0,000 & \\
\hline
\end{tabular}

${ }^{\star}$ Correlation is significant at the 0.05 level (2-tailed).

${ }^{* *}$ Correlation is significant at the 0.01 level (2-tailed).

Fonte: ns. elaborazione 
I risultati evidenziano una correlazione tra la soddisfazione e i comportamenti intenzionali, essendo la significatività inferiore a 0,05 (margine di errore del $5 \%$ ) rispetto all'intenzione d'acquisto e inferiore a 0,01 (margine di errore dell' $1 \%$ ) rispetto al passaparola. Si osserva, quindi, che ad un'elevata soddisfazione corrisponde una altrettanto elevata: i) intenzione di acquisto; ii) intenzione di effettuare un passaparola positivo. Possiamo altresì rilevare una correlazione positiva tra i singoli comportamenti intenzionali.

\subsection{La soddisfazione per i "Top Brand of the mind" e le conseguenze comportamentali}

Un approfondimento ha riguardato la misurazione della soddisfazione con riguardo ai principali (per frequenza di risposte) "Top brand of the mind" rilevati, con il fine di osservare i comportamenti intenzionali posti in essere dal consumatore che manifesta fedeltà nei confronti di un marchio. Dai dati ricavati è risultato che il $67 \%$ del campione indica il proprio "Top Brand of the mind". Focalizzando l'analisi su tale parte del campione, i marchi citati con maggiore frequenza risultano essere: Levi's (25\%), Meltin'pot (22\%) e Diesel (13\%).

La soddisfazione manifestata nei confronti di tali brand è pari a 3.86, inferiore al dato relativo all'intero campione che è pari, si rammenta, a 4,13.

Con la figura 3 si evidenziano i risultati ottenuti rilevando i valori medi di soddisfazione rispetto a ciascun item considerato e la deviazione standard.

Fig. 3: La misurazione della soddisfazione per i "Top Brand of the mind"

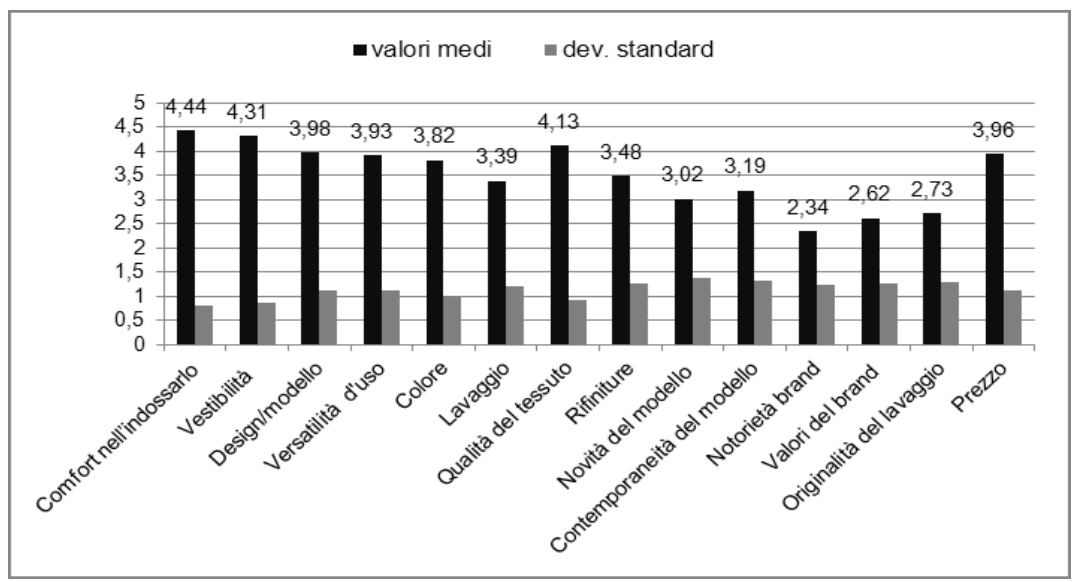

Fonte: ns. elaborazione

Rispetto ai dati inerenti l'intero campione, nel caso dei "Top brand of the mind" si rileva una maggiore attenzione alla qualità del tessuto ed al prezzo. Quest'ultimo 
rilievo evidenzia il ruolo espletato da tale variabile anche nel caso di prodotti differenziati.

La tabella 2 evidenzia i risultati dell'analisi di correlazione effettuata tra la soddisfazione media per ciascun brand e i comportamenti intenzionali.

Tab. 2: L'analisi di correlazione di Pearson (Chi Square Test) tra la soddisfazione e i comportamenti intenzionali per i "Top brand of the mind"

\begin{tabular}{|c|c|c|c|}
\hline & & Intenzione di riacquisto del brand & Passaparola \\
\hline Levi's & $\begin{array}{l}\text { Pearson Correlation } \\
\text { Sig. (2-tailed) }\end{array}$ & $\begin{array}{r}-0,007 \\
0,960\end{array}$ & $\begin{array}{c}-0,035 \\
0,808\end{array}$ \\
\hline Diesel & $\begin{array}{l}\text { Pearson Correlation } \\
\text { Sig. (2-tailed) }\end{array}$ & $\begin{array}{l}-0,111 \\
0,581\end{array}$ & $\begin{array}{l}0,262 \\
0,197\end{array}$ \\
\hline Meltin'Pot & $\begin{array}{l}\text { Pearson Correlation } \\
\text { Sig. (2-tailed) }\end{array}$ & $\begin{array}{l}0,303^{*} \\
0,046\end{array}$ & $\begin{array}{c}0,524^{* *} \\
0,000\end{array}$ \\
\hline
\end{tabular}

${ }^{*}$ Correlation is significant at the 0.05 level (2-tailed).

${ }^{* *}$ Correlation is significant at the 0.01 level (2-tailed).

Fonte: ns. elaborazione

Solo Meltin'Pot evidenzia una correlazione tra la soddisfazione percepita dal consumatore e le variabili "intenzione di riacquisto del brand" e "passaparola"; da rilevare l'elevata significatività, oltre che il valore interessante, della correlazione esistente, per il brand appena citato, tra la soddisfazione e il "passaparola". Per gli altri due brand i comportamenti intenzionali conseguenti al consumo del prodotto non sono legati alla soddisfazione percepita.

La tabella 3 rileva una significativa (in tutti i casi inferiore a 0,01 ) ed importante correlazione tra l'intenzione di acquisto e la probabilità di acquistare il brand più apprezzato.

Tab. 3: L'analisi di correlazione di Pearson (Chi Square Test) tra la generica intenzione di acquisto e l'intenzione di acquistare il "Top brand of the mind"

\begin{tabular}{|c|c|c|}
\hline & & Intenzione di riacquisto del brand \\
\hline \multirow{2}{*}{ Levi's } & Pearson Correlation & $0,622^{* *}$ \\
\hline & Sig. (2-tailed) & 0,000 \\
\hline Diesel & Pearson Correlation & $0,590^{* *}$ \\
\hline & Sig. (2-tailed) & 0,001 \\
\hline \multirow{2}{*}{ Meltin'Pot } & Pearson Correlation & $0,681^{* *}$ \\
\hline
\end{tabular}

${ }^{*}$ Correlation is significant at the 0.05 level (2-tailed).

${ }^{\star *}$ Correlation is significant at the 0.01 level (2-tailed).

Fonte: ns. elaborazione

Tale evidenza conferma che, nel caso di brand loyalty, la volontà di reiterare l'acquisto genera la specifica intenzione di riacquistare il "Top Brand of the mind". 


\subsection{Gli strumenti di supporto per l'interpretazione della soddisfazione e le} decisioni strategiche in tema di customer satisfaction

Il modello concettuale propone, in aggiunta alla misurazione della soddisfazione degli item individuati, l'utilizzo di strumenti di analisi che consentano di effettuare un approfondimento finalizzato ad una migliore interpretazione dei dati.

Ciò sia in caso di scostamenti negativi tra il dato rilevato e le attese, che per supportare l'adozione di azioni di miglioramento customer satisfaction.

Nel presente lavoro sono stati oggetto di approfondimento: i) l'indicatore di soddisfazione medio ponderato con l'importanza rilevata per ciascun item in fase di scelta del prodotto; ii) la matrice importanza/soddisfazione (nella versione proposta da Tongue e Moore, 2007).

Con tale finalità, nel questionario utilizzato è stata inserita una domanda con cui, con una scala normale da 1 a 5 , si chiedeva di indicare l'importanza attribuita ai predetti 14 item nella scelta di un jeans.

Sui dati rilevati è stata effettuata un'analisi di regressione lineare multipla, utilizzando l'intenzione di acquisto come variabile dipendente e l'insieme dei caratteri del prodotto quale variabile indipendente ${ }^{2}$. L'indice di determinazione $\mathrm{R}^{2}$ (che misura la bontà di adattamento del modello) è pari a 0,752 , con ciò significando che il modello si adatta bene a rappresentare i dati reali.

Il livello di significatività associato alla statistica test $t^{3}$, utilizzata per testare l'esistenza di un legame lineare tra la variabile dipendente e la variabile indipendente "caratteri", presenta un valore molto basso (inferiore a 0,05), confermando il legame esistente tra l'importanza attribuita ai predetti attributi e l'intenzione d'acquisto del consumatore.

2 La regressione è stata effettuata con il metodo "ANOVA" (analisi della varianza), modello lineare avente come obiettivo principale quello di identificare le fonti della variabilità (varianza) di una variabile dipendente d'interesse. Nello specifico, tale tecnica permette di studiare gli effetti di 2 o più variabili indipendenti (di natura qualitativa e che quindi formano dei gruppi) su una variabile dipendente (di natura quantitativa).

3 La statistica $t$ è usata per testare l'ipotesi "nulla" di assenza di un legame lineare tra una variabile indipendente e la variabile dipendente, ovvero che un coefficiente di regressione sia pari a zero. Il livello di significatività del test $t$ (o $p$-value, colonna Sign., è la probabilità molto bassa di commettere un errore, ritenendo non nullo il coefficiente di regressione) consente di decidere se rifiutare o meno la suddetta ipotesi nulla, in base al livello di confidenza considerato (nel nostro caso abbiamo considerato un livello di confidenza del 95\%). Se il livello di significatività osservato è abbastanza basso (come nel nostro caso) allora l'ipotesi nulla è rifiutata. 
Tab. 4: L'analisi di regressione lineare tra l'intenzione d'acquisto e i caratteri del prodotto

\begin{tabular}{|c|c|c|c|c|c|}
\hline \multirow{2}{*}{ Modello } & \multicolumn{2}{|c|}{$\begin{array}{c}\text { Coefficienti non } \\
\text { standardizzati }\end{array}$} & \multirow{2}{*}{$\begin{array}{c}\begin{array}{c}\text { Coefficienti } \\
\text { standardizzati }\end{array} \\
\text { Beta }\end{array}$} & \multirow{2}{*}{$\mathrm{t}$} & \multirow{2}{*}{ Sign. } \\
\hline & $B$ & $\begin{array}{c}\text { Errore } \\
\text { Standard }\end{array}$ & & & \\
\hline Caratteri & 0,042 & 0,009 & 0,584 & 4,747 & 0,000 \\
\hline
\end{tabular}

a. Variabile Dipendente: intenzione d'acquisto.

b. Regressione Lineare attraverso l'origine.

Fonte: ns. elaborazione.

Avendo appurato il legame esistente tra l'intenzione d'acquisto del prodotto e le variabili, si è verificata l'importanza assegnata dagli intervistati ai singoli caratteri, che ha evidenziato i risultati riportati nella figura 2.

Fig. 4: Gli attributi determinanti l'acquisto del jeans

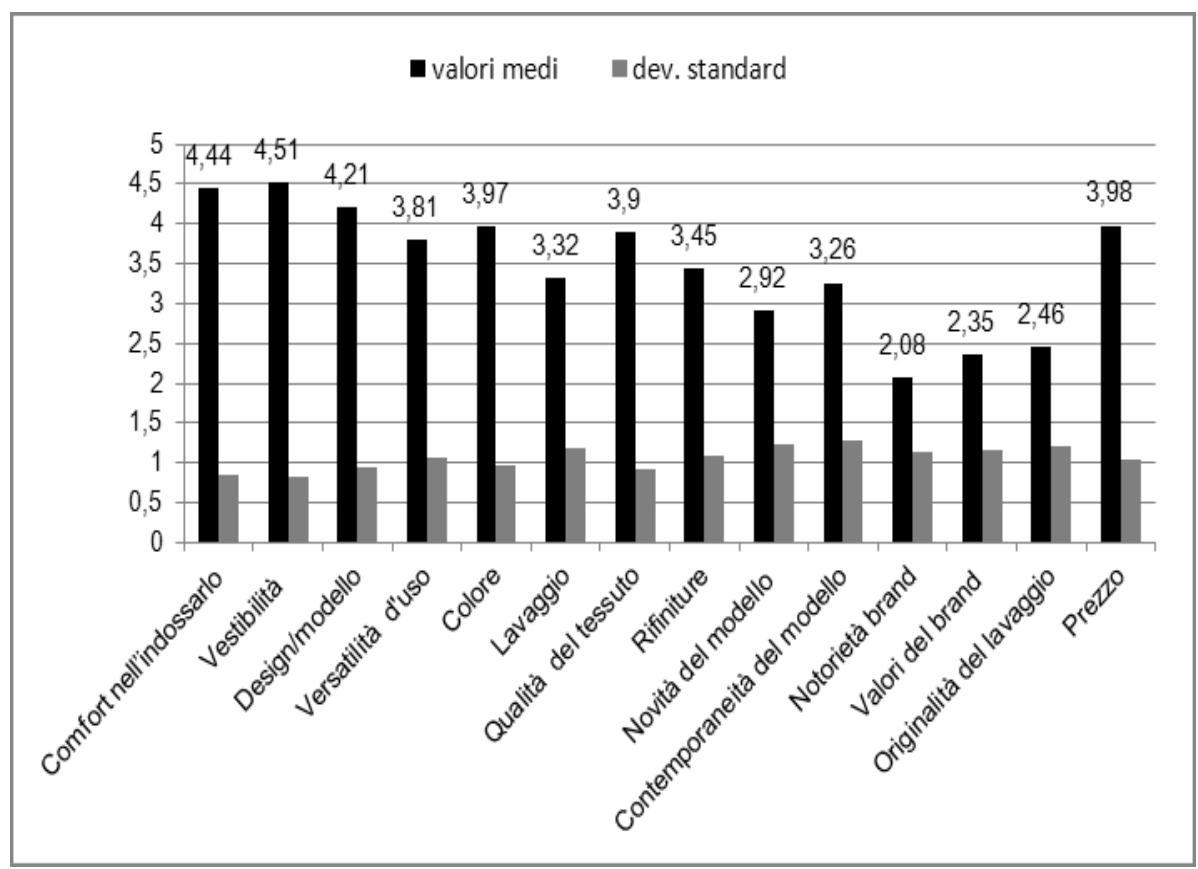

Fonte: ns. elaborazione. 
"Comfort", "Vestibilità" e "Design/Modello" rappresentano i primi attributi per importanza assegnata. Da non trascurare il ruolo esercitato dall'item "prezzo", in considerazione anche della soddisfazione che lo stesso genera anche negli acquisti dei "Top Brand of the mind".

In seguito a tale preliminare analisi, si è proceduto con la verifica dell'indicatore medio ponderato e con la costruzione della matrice delle priorità. Ciò inizialmente lo si è fatto per l'intero campione e poi sui dati ottenuti per i "Top brand of the mind".

L'indicatore di soddisfazione media ponderata ${ }^{4}$ è risultato essere pari a 3,71 , inferiore al valore fatto rilevare dalla verifica dell'indice di soddisfazione medio semplice, pari a 4,13. Tale evidenza dimostra che il livello di soddisfazione perseguito, sebbene positivo, non rispetta le aspettative (importanza) manifestate dal consumatore. Quindi, vi sono dei caratteri il cui livello di importanza non trova adeguata soddisfazione nell'offerta acquisita.

La costruzione della matrice importanza/soddisfazione ha consentito di evidenziare i gap di soddisfazione per ciascun attributo e, in considerazione della collocazione degli stessi all'interno della matrice, di procedere con opportune valutazioni sulle priorità di intervento al fine di investire le risorse disponibili per il miglioramento della competitività dell'offerta proposta al mercato ${ }^{5}$.

$4 \quad$ IDS medio ponderato $=\underline{(\operatorname{IDS} \mathrm{a} * \text { IDI } \mathrm{a})+(\operatorname{IDS} \mathrm{b} * \operatorname{IDI} \mathrm{b})+\ldots}$

$$
(\text { IDI } a)+(\text { IDI } b)+\ldots
$$

5 Tale matrice individua quattro aree che richiedono differenti priorità di attenzione ed intervento, di seguito descritte:

1. Area delle criticità (Concentrate management here, in alto a sinistra): raggruppa gli attributi che manifestano una importanza elevata e una bassa soddisfazione, ragione per cui necessitano di interventi prioritari, dato appunto il livello di importanza assegnato agli stessi dai consumatori;

2. Area del monitoraggio (low priority for managers, in basso a sinistra): individua gli elementi che evidenziano una bassa importanza, cui corrisponde anche una bassa soddisfazione del consumatore e quindi costituiscono i punti di debolezza marginali, con necessità di interventi di priorità secondaria;

3. Area della competitività (Keep up the good work, in alto a destra): identifica i punti di forza sostanziali del prodotto in quanto comprende gli attributi definiti importanti dal consumatore nella scelta del prodotto e per i quali la soddisfazione è altrettanto elevata; per tale motivo è utile che si proceda con un monitoraggio continuo di tali caratteri, che possa essere utile per adottare strategie di consolidamento delle performance;

4. Area delle illusioni (Possible overkill, in basso a destra): costituita da attributi a cui il consumatore assegna un'importanza marginale nella scelta del prodotto, sebbene lo stesso giudichi elevata la soddisfazione percepita; pertanto, occorre monitorare gli stessi per evitare di impiegare risorse per lo sviluppo di caratteri del prodotto non apprezzati dal consumatore. 


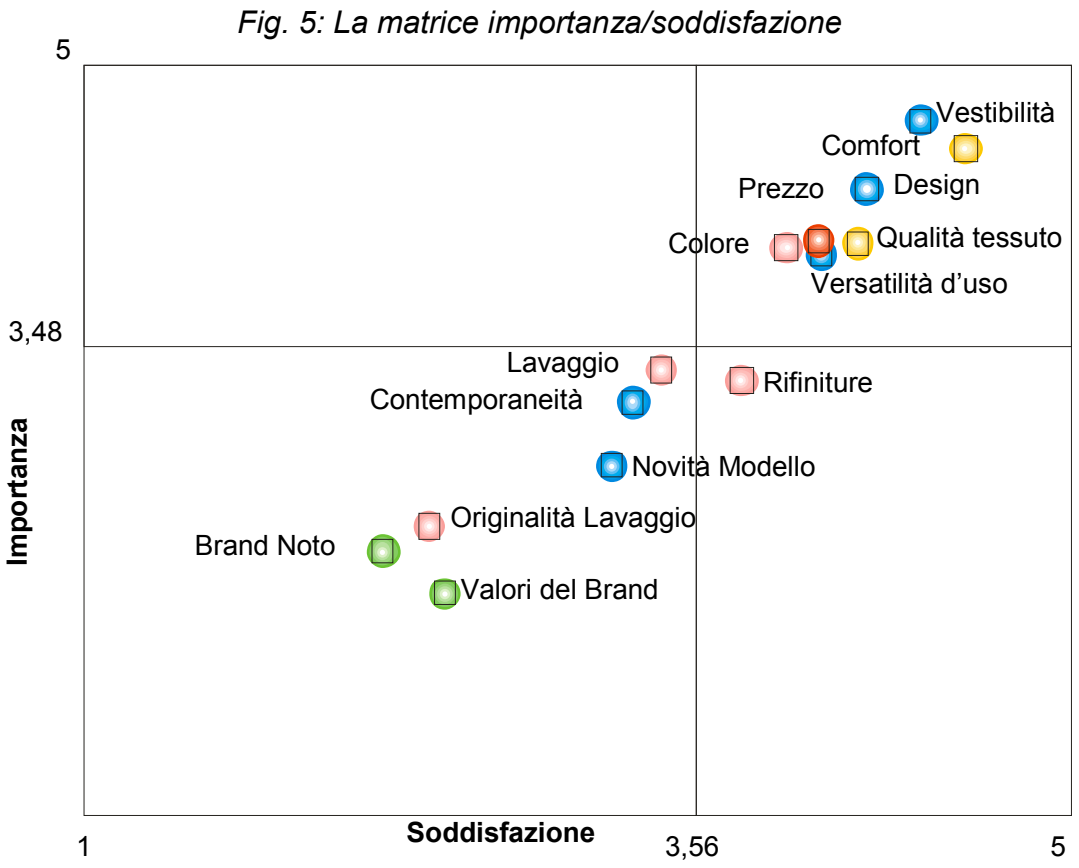

Fonte: ns. elaborazione

La figura 5 evidenzia: a) l'assenza di item nell'area delle priorità; b) la collocazione dei caratteri inerenti il brand (valori e notorietà) nell'area del monitoraggio; c) la presenza dell'item "rifiniture" nell'area delle illusioni.

Per ciò che concerne i "Top Brand of the mind", l'indicatore di soddisfazione media ponderata ${ }^{6}$ è risultato essere pari a 3,66 , inferiore al valore rilevato dalla verifica dell'indice di soddisfazione medio semplice, pari complessivamente per i tre brand a 3,86. La tabella 5 riporta il confronto tra i due indicatori per ciascun brand.

Tab. 5: L'indice medio di soddisfazione e medio ponderato per ciascun "Top brand of the mind"

\begin{tabular}{|l|c|c|}
\hline Brand & Indice medio & Indice medio ponderato \\
\hline Levi's & 3.84 & 3,44 \\
\hline Diesel & 4.00 & 3,88 \\
\hline Meltin Pot & 3.80 & 3,80 \\
\hline
\end{tabular}

Fonte: ns. elaborazione

6 IDS medio ponderato $=(\underline{\text { IDS a } . ~ I D I ~ a ~})+(\operatorname{IDS} \mathrm{b}$. IDI b $)+\ldots$ $($ IDI $a)+($ IDI $b)+\ldots$ 
Solo il brand Meltin'Pot evidenzia un indice medio ponderato in linea con quello semplice. Per gli altri brand vi sono item la cui soddisfazione non appaga l'importanza attribuita dal consumatore in fase di acquisto.

La matrice importanza-soddisfazione (fig. 6), costruita per ciascun "Top Brand of the mind", consente di chiarire, tra le altre, il ruolo espletato da ciascun item nella percezione della soddisfazione complessiva. L'utilità delle applicazioni successive conferma i rilievi posti dalla letteratura (da ultimo, Chen, 2014) in merito al fatto che non si ritrovano applicazioni della matrice per un'analisi tra competitors.

Fig. 6: La matrice importanza-soddisfazione per ciascun "Top brand of the mind"

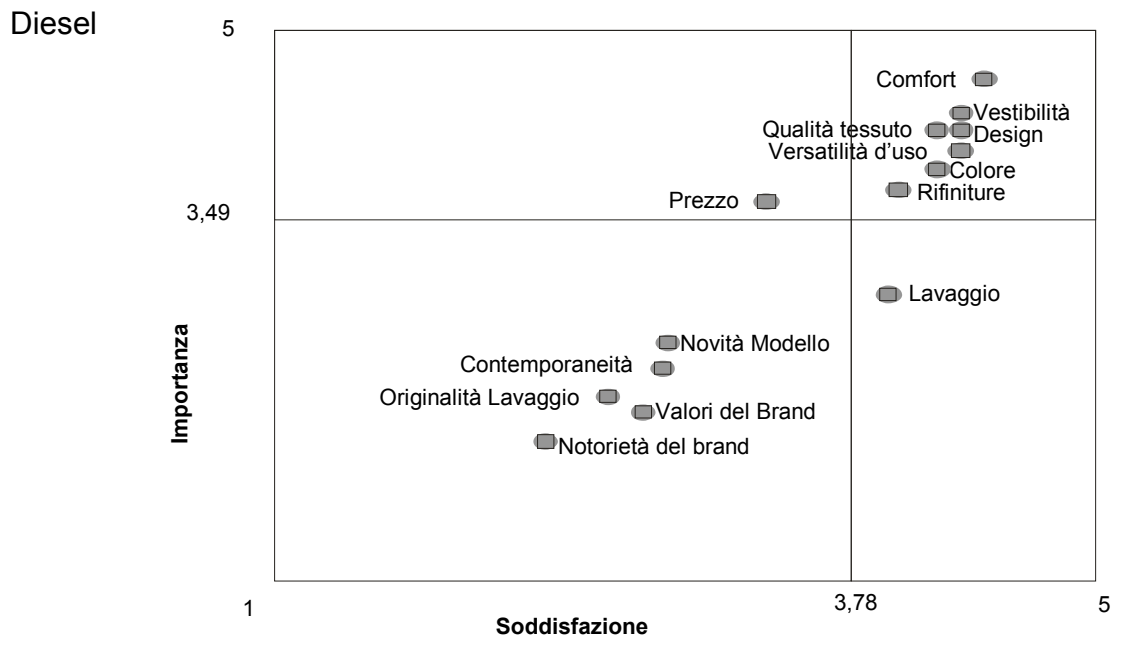

Meltin'Pot

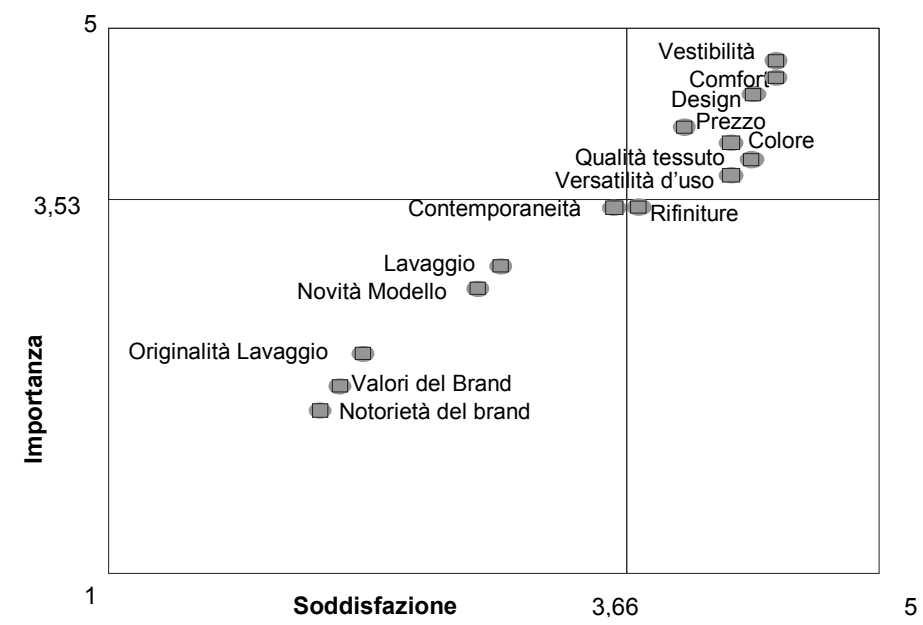


Levi's

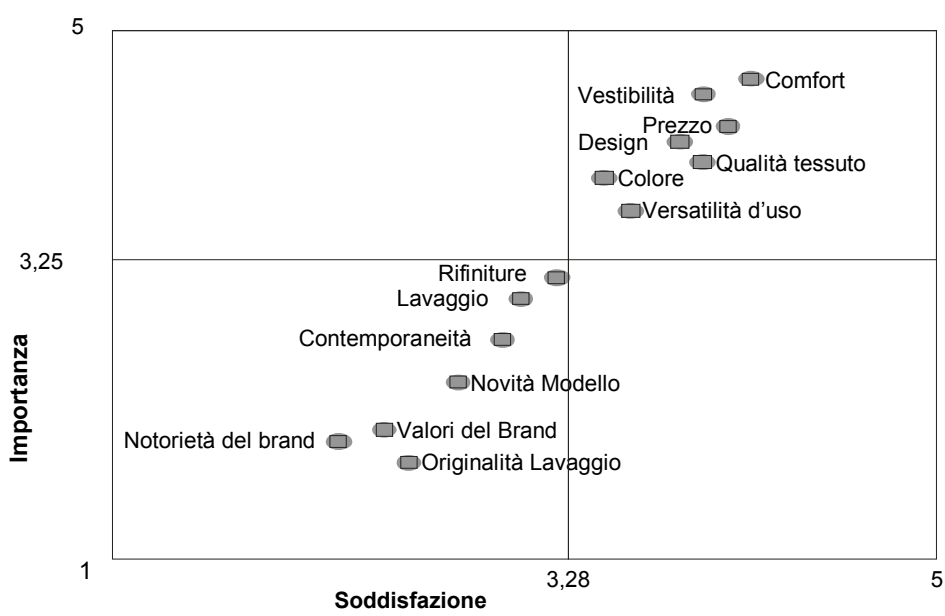

Fonte: ns. elaborazione

Rispetto al dato complessivo, la rilevazione inerente $\mathrm{i}$ singoli brand ha evidenziato delle peculiarità che offrono utili spunti di riflessione per il management. In particolare, i consumatori abituali di Diesel considerano il lavaggio poco importante nella scelta del brand, ma gli attribuiscono una valutazione di soddisfazione alta; ovverosia, apprezzano gli sforzi ma basterebbe anche meno per quell'attributo. Stessa valutazione è espressa dai consumatori di Meltin'Pot per l'item "rifiniture". Particolare attenzione richiede, invece, per Diesel la strategia di prezzo, essendo lo stesso attributo ricaduto nell'area delle priorità di intervento; in tale circostanza, per il management si richiedono iniziative tese a ridurre il prezzo di vendita 0 , in alternativa, a farne percepire la coerenza rispetto all'offerta proposta.

Per tutti e tre i brand vale la considerazione fatta in precedenza rispetto agli attributi "notorietà del brand" e "valori del brand", presenti nell'area del monitoraggio, che richiede una priorità di intervento secondaria. Una spiegazione può derivare dalla circostanza che, generalmente, in presenza di brand loyalty il consumatore non guarda a soluzioni alternative e quindi difficilmente valuta altri marchi (e quindi i caratteri degli stessi).

Si consideri, infine, l'utilità di osservare la differente posizione rilevata per ciascun item all'interno dell'area della competitività; il differente connubio importanza-soddisfazione può indirizzare il management ad intraprendere differenti azioni anche per le variabili rientranti in una stessa area.

\section{Conclusioni ed implicazioni manageriali}

Le relazioni tra imprese e consumatori evidenziano un carattere diadico, frutto di un percorso dinamico, articolato in azioni e reazioni poste in essere dai soggetti con 
il fine di perseguire i propri obiettivi. Pertanto, la conoscenza del comportamento del consumatore e il monitoraggio della sua soddisfazione rappresentano elementi imprescindibili su cui fondare la pianificazione strategica delle imprese, in particolare nei contesti dinamici e vocati alla differenziazione quali quello del jeans. Monitorare costantemente la soddisfazione ed i comportamenti che da essa ne possono scaturire rappresenta uno step di analisi fondamentale per la genesi ed il mantenimento della competitività.

Il percorso adottato nel presente lavoro consente di rispondere ad una duplice necessità del management: a) disporre di indicatori di sintesi della soddisfazione del consumatore; b) acquisire informazioni analitiche e esaustive dello stesso costrutto, utili per la pianificazione strategica.

Il primo obiettivo raggiunto, infatti, è stato quello di individuare le determinanti della soddisfazione del consumatore di jeans.

Trattandosi di un settore in cui vi è un spiccato orientamento all'adozione di politiche di differenziazione, si è ritenuto utile effettuare uno specifico approfondimento sul ruolo che il brand assume tanto nella misurazione della stessa soddisfazione che negli atteggiamenti conseguenti al consumo posti in essere dal consumatore. Infatti, il secondo obiettivo è stato proprio quello di verificare e misurare (ove esistenti) i legami tra il costrutto della soddisfazione e i comportamenti che da essa ne possono derivare.

I dati inerenti l'intero campione hanno evidenziato la presenza di un legame tra la soddisfazione del consumatore e i comportamenti conseguenti all'esperienza di consumo. Una maggiore correlazione è stata rilevata tra la soddisfazione e il "passaparola", ovverosia quel comportamento con cui il consumatore diffonde informazioni (positive o negative, a seconda del giudizio espresso) su un prodotto o su un'impresa in seguito ad una o più esperienze di consumo. Tale attività è per sua natura difficilmente controllabile dalle imprese, a maggior ragione nel contesto attuale, in cui internet e gli strumenti forniti dal web 2.0 rappresentano un elemento catalizzatore della velocità di diffusione delle informazioni. In considerazione delle potenzialità del "passaparola" - ovverosia della velocità con cui si veicolano le informazioni e della sua efficacia in termini di impatto sulle scelte - (Romaniuk, 2007)- nonché della crescente diffusione dei nuovi mezzi e strumenti della social media communication, diviene indispensabile per il brand management fruire di tali potenzialità per fornire la risposta ad un contesto comunicativo co-gestito da e per i clienti (Greenberg, 2009), e per tale via incrementare la conoscenza di questi ultimi, di acquisirne nuovi e, di conseguenza, personalizzare la relazione. In ipotesi di passaparola negativo, gli strumenti appena citati, nell'ambito di una pianificata azione di gestione dei reclami, possono consentire in modo rapido ed efficace di ripristinare la relazione con clienti insoddisfatti ed evitare reazioni che possono generare effetti negativi (abbandono, passaparola negativo, reazioni vendicative) su fatturato e immagine aziendale.

Per ciò che concerne i "Top brand of the mind" si è osservata l'assenza di legame tra le predette variabili per due dei tre brand. Ciò a riprova del fatto che $\mathrm{i}$ comportamenti intenzionali non sempre sono determinati dalla soddisfazione 
percepita dal consumatore. In particolare, la reiterazione dell'acquisto può essere motivata da particolari stati emozionali con cui si vive un'esperienza di consumo, altrimenti denominati consumer delight (Keiningham e Vavra, 2001), o dalla fedeltà al prodotto o al brand (brand loyalty), che può raggiungere valori tali da determinare uno stato di "tensione all'azione" nel consumatore (Oliver, 1999), il quale reitera il proprio comportamento d'acquisto in modo abitudinario, senza impegnare risorse nella scelta e quindi evitando valutazioni di ogni genere.

In tali circostanze il management può: i) adottare strategie che siano in grado di conservare la brand loyalty, pianificando azioni volte a generare stati emotivi e di coinvolgimento tali da conservare il grado di fedeltà maturato; il riferimento specifico è a campagne promozionali in grado di corroborare la brand image o a specifiche azioni tendenti a stimolare la "gratitudine" del consumatore (ovverosia lo stato affettivo che, nel rapporto di scambio, si matura per effetto della percezione che la controparte ha intrapreso deliberatamente azioni per migliorare il benessere; Raggio e Folse, 2009; ii) lavorare per migliorare la soddisfazione rispetto agli specifici attributi a cui il consumatore ha attribuito livelli di importanza elevata nelle sue scelte di acquisto.

Nella scelta delle alternative strategiche disponibili è di estrema utilità il ricorso a strumenti di analisi, quali la matrice importanza-soddisfazione, in grado di fornire un'interpretazione analitica dei risultati ottenuti e quindi fornire informazioni indispensabili per il management (così come emerso dal lavoro). Nel contesto attuale risulta indispensabile per il management adottare modelli di customer satisfaction che siano in grado di monitorare in modo completo l'atteggiamento del consumatore.

\section{Bibliografia}

AAKER D.A. (1991), Managing Brand Equity, Free Press, San Francisco.

AAKER D.A. (2001), Strategic Market Management, 6th edition, John Wiley \&' Sons, New York.

AUTY S., ELLIOT R. (1998), "Fashion involvement, self-monitoring and the meaning of brands", Journal of Product and Brand Management, vol. 7, n. 2, pp. 109-123.

AYBENIZ AKDENIZ A.R. (2012), "Effect of Perceived values on the brand preference and the purchase intention", European Scientific Journal August edition, vol. 8, n. 17 pp. $1-17$.

BACCARANI C., GOLINELLI G.M., (1992), "L'impresa inesistente: le relazioni tra immagine e strategia", Sinergie, n. 29, pp. 137-147.

BETTMAN J.R. (1979), An information processing theory of consumer choice, Reading, MA Addison-Wesley.

BRENNAN L., MAVONDO F. (2000), “Involvement: An Unfinished Story?", Proceedings of the Australian and New Zealand Marketing Academy Conference (ANZMAC 2000) on "Visionary Marketing for the 21 st Century: Facing the Challenge", Gold Coast, Queensland, Australia, 28 November-01 December 2000, pp. 133-137. 
BRUNNER T., STÖCKLIN M., OPWIS K. (2008), "Satisfaction, image and loyalty: new versus experienced customers", European Journal of Marketing, vol. 42, n. 9/10, pp. $1095-1105$.

BUSACCA B. (2000), Il valore della marca tra postfordismo ed economia digitale, Egea, Milano.

BUSACCA B., BERTOLI G., (2008), Valore per il cliente, soddisfazione, fedeltà, Egea, Milano.

CANTONE L., RISITANO M. (2006), "Il ruolo delle basi cognitive del valore di marca nella determinazione del comportamento di acquisto del consumatore", Sinergie, n. 70, pp. 219-247.

CASTALDO S. (2002), Fiducia e relazioni di mercato, Il Mulino, Bologna.

CHEN K.Y. (2014), "Improving importance-performance analysis: The role of the zone of tolerance and competitor performance. The case of Taiwan's hot spring hotels" Tourism Management, vol. 40, pp. 260-272.

CHI C.G., QU H. (2008), "Examining the structural relationships of destination image, tourist satisfaction and destination loyalty: An integrated approach", Tourism Management, vol. 29 , n. 4 , pp. 624-636.

CHOWDRAY U. (2002), "Does price reflect emotional, structural or performance quality?", International Journal of Consumer Studies, vol. 26, n. 2, pp. 128-133.

CORBETTA P., GASPERONI G., PISATI M. (2001), Statistica per la ricerca sociale, Il Mulino, Bologna.

COSTABILE M. (1996), Misurare il valore per il cliente, Utet, Torino.

COSTABILE M., (2000), "Un modello dinamico di customer loyalty", Finanza, Marketing e Produzione, vol. 18, n. 3, pp. 3-36.

COSTABILE M., RAIMONDO M.A., MICELI G. (2004), "Un modello dinamico di customer loyalty: evidenze empiriche da un analisi intergruppo con modelli di equazioni strutturali”, Finanza, marketing e produzione, vol. XXII, n. 4, pp. 41-68.

DALLI D., ROMANI S. (2003), Il comportamento del consumatore, FrancoAngeli, Milano.

DE VELLIS R.F. (1991), Scale Development. Theory and Applications, London Sage.

DELONG M. (1998), The Way We Look: Dress and Aesthetics, 2nd ed., Fairchild, New York, NY.

DELONG M., LABAT K., NELSON N., KOH A., KIM Y. (2002), "Global products, global markets: Jeans in Korea and the United States", Clothing and Textiles Research Journal, vol. 20, n. 4, pp. 238-245.

DIMITRIADES Z.S. (2006), "Customer satisfaction, loyalty and commitment in service organizations-Some evidence from Greece", Management Research News, vol. 29, n. 12, pp. 782-800.

EAST R. (1997), Consumer behaviour: Advances and applications in marketing, PrenticeHall, London.

EICHER J. B., EVENSON S. L., LUTZ H. A. (2000). The visible self: Global perspectives on dress, culture, and society. (2nd ed). New York: Fairchild.

ENGEL J.F., BLACKWELL R.D., MINIARD P.W. (1995), Consumer Behavior, $8^{\text {th }}$, Chicago: the Dryden Press.

ENOCHS C. (1993), "Jeans as material culture: a comparison of male and female college students in the mid-western United States of America", unpublished master's thesis, University of Minnesota, St Paul.

FAULLANT R., MATZLER K. (2008), "The impact of satisfaction and image on loyalty: the case of Alpineski resorts", Managing Service Quality, vol. 18, n. 2, pp. 163-178. 
FISHBEIN M., AJZEN I. (1975), Belief, Attitude, Intention, and Behavior: An Introduction to Theory and Researc. MA: Addison-Wesley.

FORNELL C., MITHAS S., MORGESON III F.V., KRISHNAN M.S. (2006), "Customer satisfaction and stock prices: High returns, low risk", Journal of Marketing, vol. 70, n. 1, pp. 3-14

FU C.S., WU W.Y. (2013), "Means-end matrix and deduction in consumption behavior research", European Journal of Research Methods for the Behavioral and Social Sciences, vol. 9, n. 2, pp. 54-68.

FULLERTON G. (2005), Predictors of trauma response in sexually abused children: The roles of resiliency and protective factors, Unpublished master's thesis, University of Houston, Houston, Texas.

FULLERTON G. (2003), "When Does Commitment Lead to Loyalty?," Journal of Service Research, vol. 5, n. 4, pp. 333-344.

GIESE J.L., COTE J.A. (2000), "Defining Customer Satisfaction", Academy of Marketing Science Review, vol. 2000, n. 1, pp. 1-59.

GREENBERG P. (2010), "The impact of CRM 2.0 on customer insight", Journal of Business \& Industrial Marketing, vol. 25, n. 6, pp. 410-419.

GUIDO G., BASSI F., PELUSO A.M. (2010), La soddisfazione del consumatore, Franco Angeli, Milano.

HAUSKNECTH D.R. (1988), "Emotional Measures of Satisfaction/Dissatisfaction", Journal of Consumer Satisfaction, Dissatisfaction and Complaining Behavior, vol. 3, pp. 1-11.

HEILMAN C.M., BOWMAN D., WRIGHT G.P. (2000), "The Evolution of Brand Preferences and Choice Behaviors of Consumers New to a Market", Journal of Marketing Research, vol. 37, n. 2, pp. 139-155.

HERBST F., BURGER C. (2002), "Attributes used by consumers when purchasing a fashion product: A conjoint analysis approach", Journal of Family Ecology and Consumer Sciences, vol. 30, pp. 40-45.

HIRSCH E.W.A. (2011), "Customer Satisfaction in a High-technology Business-to-Business Context", http://www.duo.uio.no/

HOLBROOK M.B., HIRSCHMAN E.C., "The experiential aspects of consumption: consumer fantasies, feelings and fun", Journal of Consumer research, vol. 9, September 1982, pp. 132- 140.

HOWARD J.A., SHETH J.N. (1969), The Theory of Buyer Behavior, John Wiley and Sons. New York.

JIN B., PARK J.Y., KIM H.S.(2010), "What makes online community members commit? A social exchange perspective", Behaviour \& Information Technology, vol. 29, n. 6, pp. 587-599.

JIN B., KANG J.H (2011), "Purchase intention of Chinese consumers toward a US apparel brand: a test of acomposite behavior intention model", Journal of Consumer Marketing, vol. 28, n. 3, pp. 187-199.

JOHNS N. (2001), "Importance-performance analysis using the profile accumulation technique", The Service Industries Journal, vol. 21n. 3, pp. 49-63.

KAHLE R.L. (1983), Social values and social change: Adaptation to life in America, N. Y. Praeger, New York.

KEININGHAM T., VAVRA T., (2001), The Customer Delight Principle, McGraw-Hill, New York

KELLER K.L. (1993), "Conceptualising, Measuring and Managing Customer-Based Brand Equity”, Journal of Marketing, vol. 57, n. 1, pp. 1-22. 
KELLER K.L. (2003), Strategic Brand Management: Building, Measuring and Managing Brand Equity, second edition, Prentice Hall. New Jersey.

KOTLER P. (1991), Marketing Management (7th ed). Englewood Cliffs, Prentice- Hall New Jersey.

LABARBERA P. A., MAZURSKY D.,(1983), "A Longitudinal Assessment of Consumer Satisfaction/Dissatisfaction: The Dynamic Aspect of the Cognitive Process", Journal of Marketing Research, n. 20 (November), pp. 393-404.

LAMBIN J.J. (2012), Market-driven Management, McGraw-Hill, Milano.

LEVENBURG N.M., MAGAL S.R., (2005), "Using Importance-Performance Analysis to Evaluate E-Business Strategies among Small Businesses", e-Service Journal, vol. 3, n. 3 , pp. $29-48$.

LEE C. (1990), "Modifying an American consumer behavior model for consumers in Confucian culture: the case of Fishbein behavioral intention model", Journal of International Consumer Marketing, vol. 3, n. 1, pp. 27-50.

LUO X., HOMBURG C. (2007), "Neglected Outcomes of Customer Satisfaction”, Journal of Marketing, vol. 71, n. 2, pp. 133-149.

LUO X., HOMBURG C., WIESEKE J. (2010), "Customer Satisfaction, Analyst Stock Recommendations, and Firm Value", Journal of Marketing Research, vol. XLVII, pp. $1041-1058$.

MAIZZA A. (2013), Management d'impresa e strategie competitive, Cacucci, Bari.

MARTILLA J., JAMES J. (1977), 'Importance-Performance Analysis', Journal of Marketing, vol. 41, n. 1, pp. 77-79.

MATTIACCI A., MASON M.C. (2009), "Metodi e tecniche della ricerca di marketing, in Cristini G., Il Marketing, Il Sole 24 Ore, Milano.

MATZLER K., BAILOM F., HINTERHUBER H.H., RENZL B., PICHLER J. (2004), "The asymmetric relationship between attribute-level performance and overall customer satisfaction: a reconsideration of the importance-performance analysis", Industrial Marketing Management, vol. 33, n. 4, pp. 271-277.

MATZLER K., SAUERWEIN L., HEISCHMIDT K.A. (2003), "Importance e performance analysis revisited: the role of the factor structure of customer satisfaction", The Service Industries Journal, vol. 23, n. 2, pp. 112-129.

MAURI A.G. (2002), Le prestazioni dell'impresa come comunicazione di fatto e il ruolo del passaparola", Sinergie, n. 59, pp. 147-159.

MCMULLAN R., GILMORE A. (2008), "Customer loyalty: an empirical study", European Journal of Marketing, vol. 42, n. 9-10, pp. 1084-1094.

MOLTENI L., TROILO G. (2003), Ricerche di marketing, Milano, McGraw-Hill.

OLIVER R.L. (1997), Satisfaction: A Behavioral Perspective on the Consumer, New York: The McGraw-Hill Companies, Inc.

OLIVER R.L. (1980), "A Cognitive Model of the Antecedents and consequences of satisfaction decision”, Journal of Marketing Research, vol. XVII, November, pp. 460469.

OLIVER R.L. (1999), “Whence customer loyalty?”, Journal of Marketing, vol. 63, n. 4, pp. 33-44.

OLIVER R.L. (2010), Satisfaction. A behavioral Perspective on the Consumer, $2^{\text {nd }}$ ed., M.E. Sharpe, Armonk, New York.

PARK W., LEE S. (1999), "A market oriented study on the wearing attitude and purchase behavior of jeans", Journal of Costume, vol. 43, pp. 109-123. 
RAGGIO R. D., FOLSE J.A.. G. (2009), "Gratitude Works: Its Impact and the Mediating Role of Affective in Driving Positive Outcomes", Journal of the Academy of Marketing Science, n. 37 (December) pp. 455-469.

RAHMAN O. (2011), "Understanding Consumers' Perceptions and Behaviors: Implications for Denim Jeans Design", Journal of Textile and Apparel, Technology and Management, vol. 7, n. 1, Spring 2011.

RAITHEL S., SARSTEDT M., SCHARF S., SCHWAIGER M., (2011), "On the value relevance of customer satisfactio, n. Multiple drivers and multiple markets”, Journal of the Academy Marketing Science, vol. 40, n. 4, pp. 509- 525.

RAUYRUEN P. , MILLER K.E. (2007), "Relationship quality as a predictor of B2B customer loyalty", Journal of business research, vol. 60, n.1, pp.21-31.

RICE C., (1993), Consumer behaviour: Behavioural aspects of marketing, ButterworthHeinemann (Oxford and Boston).

ROCKEACH M. (1973), The Nature of human values, The Free Press, N. Y.

ROMANIUK J. (2007), "Word of Mouth and the viewing of television programs", Journal of Advertising Research, vol. 47, n. 4, pp. 462-471.

RUSSEL C.A., NORMAN A.T., HECKLER S.E. (2004), "The consumption of television programming: Development and validation of the connectedness scale", Journal of Consumer Research, vol. 31, n. 1, pp. 150-161.

SAMPSON S.E., SHOWALTER M.J. (1999), "The performance - importance response function: Observations and implications", The Service Industries Journal, vol. 19, n. 3 , pp. $1-25$

SOLOMON M.R. (2004), Consumer Behavior. Buying, Having, and Being, Pearson Prenctice Hall. Saddle River.

SPRENG R.A., MACKENZIE S.B., OLSHAVSKY R.W. (1996), "A Reexamination of the Determinants of Consumer Satisfaction", Journal of Marketing, Vol. 60, No. 3, July, pp. 15-32.

SWAN J.E., TRAWICK I.F. (1980), "Satisfaction Related to Predictive vs. Desired Expectations: A Field Study", in Hunt H.K., Day G.S. (a cura di), Refining Concepts and Measures of Consumer Satisfaction and Complaining Behavior, School of Business, Indiana University, Bloomingto, pp. 15-22.

TANESE, A., NERO, G., GRAMIGNA, A. (2003), La customer satisfaction nelle amministrazioni pubbliche, Rubettino Editore.

TONGUE J., MOORE S. A. (2007), "Importance-satisfaction analysis for marine-park hinterlands: a Western Australian case study", Tourism Management, vol. 28, pp. 768-776.

TSAI H.T., HUANG H.C., JAW Y.L., CHEN W.K. (2006), "Why on-line customers remain with a particular e-retailer: An integrative model and empirical evidence", Psychology and Marketing, vol. 23, n. 5, pp. 447-464

VICARI S. (1995), "Verso il Resource-Based Management" in Vicari S., Brand Equity, Egea.

VON WANGENHEIM, F., (2005) "Postswitiching Negative Word of Mouth", Journal of Service Research, vol. 8, n. 1, pp. 67-78

VRONTIS, D., VRONTIS, P. (2004), "Levi Strauss: an international marketing investigation", Journal of Fashion Marketing and Management, vol. 8, n. 4 pp. 389398.

WESTBROOK R. A. (1980), "Consumer Satisfaction as a Function of Personal Compretence/Efficacy", Journal of the Academy of Management Science, n. 8 (September), pp. 427-437. 
WESTBROOK R.A., OLIVER R. (1981), "Developing Better Measures of Consumer Satisfaction: Some Preliminary Results," Advances in Consumer Research, vol. 8 pp. 94-99.

WESTBROOK R.A., OLIVER R.L., (1991), "The Dimensionality of Consumption Emotion Patterns and Consumer Satisfaction”, Journal of Consumer Research, vol. 18, pp. 84-91.

WORKMAN J.E. (1988), "Trait inferences based on perceived ownership of designer, brand name, or store brand jeans", Clothing and Textiles Research Journal, vol. 6, n. 2 pp. 23-29.

WU J. (2005), "Chinese perceptions of western-branded denim jeans: a Shanghai case study", unpublished doctoral dissertation, University of Minnesota, Twin Cities, MN.

WU J., DELONG M. (2006) "Chinese perceptions of western-branded denim jeans: a Shanghai case study", Journal of Fashion Marketing and Management, vol. 10, n. 2 pp. 238-250.

YI Y., LA S. (2004), "What influences the relationship between customer satisfaction and repurchase intention? Investigating the effets of adjusted expectations and customer loyalty", Psychology and Marketing, vol. 21, n. 5, pp. 351-373.

YOUNG R. (1980), "Denim jeans: consumer preferences and manufacturers views", unpublished doctoral dissertation, Texas Woman's University, Denton, TX.

ZHANG Z., LI Y., GONG C., WU H. (2002), "Casual wear product attributes: a Chinese consumer's perspective”, Journal of Fashion Marketing and Management, vol. 6, n. 1 pp. 53-62. 
